\title{
Design Optimization of a Series Plug-in Hybrid Electric Vehicle for Real-World Driving Conditions
}

\author{
Rakesh Patil, Brian Adornato, and Zoran Filipi \\ Mechanical Engineering Department, University of Michigan, Ann Arbor
}

Copyright (C) 2010 SAE International

\section{ABSTRACT}

This paper proposes a framework to perform design optimization of a series PHEV and investigates the impact of using real-world driving inputs on final design. Real-World driving is characterized from a database of naturalistic driving generated in Field Operational Tests. The procedure utilizes Markov chains to generate synthetic drive cycles representative of real-world driving. Subsequently, PHEV optimization is performed in two steps. First the optimal battery and motor sizes to most efficiently achieve a desired All Electric Range (AER) are determined. A synthetic cycle representative of driving over a given range is used for function evaluations. Then, the optimal engine size is obtained by considering fuel economy in the charge sustaining (CS) mode. The higher power/energy demands of real-world cycles lead to PHEV designs with substantially larger batteries and engines than those developed using repetitions of the federal urban cycle (UDDS). This is a finding of high relevance for forecasting technology diffusion, consumer acceptance, and impact of PHEVs on power grid. These differences increase progressively with desired AER due to increasing energy/mile usage of real world driving with distance.

\section{INTRODUCTION}

Plug-in Hybrid Electric Vehicles (PHEV) link two major infrastructures, the transportation and the electric power grid. They offer a promise of significant reduction of fuel consumption, oil imports, and $\mathrm{CO}_{2}$ emissions by bringing other sources of energy into transportation. PHEVs are similar to conventional Hybrid Electric Vehicles (HEV) in that they utilize an electric motor and a gasoline engine as two drives to power a vehicle, but they have much larger batteries that can be charged from an electrical outlet. The electrical energy stored in the battery is used as the primary source of power on-board until the battery charge is depleted to a threshold level. This mode of powertrain operation is called the charge depleting (CD) mode or electric vehicle (EV) mode. After charge depletion to the threshold level the internal combustion engine is used to sustain the charge at this level. This is referred to as the charge sustaining mode (CS) mode. The all-electric range (AER) or the charge depleting range (CDR) of a PHEV is the distance over which the powertrain operates in EV or CD mode respectively and this has been recognized a very relevant attribute for PHEVs [1] [2] [3]. The engine fuel consumption during this mode is very low or zero. This has led to classification of PHEVs as PHEV 10 (A PHEV with 10 miles CDR/AER), PHEV 20 and so on. If pure AER is desired, a Series Hybrid Electric Vehicle configuration is particularly interesting. However, many design decisions have to be made to maximize the potential of a given configuration for a selected vehicle application.

Several design studies have been undertaken for hybrid electric and plug-in hybrid electric vehicles in the past. In [4] it is clearly acknowledged that the energy consumption of a PHEV is strongly linked to the size of its

Page 1 of 14 
powertrain components. The study in [4] considers different sets of battery and motor sizes and an optimal controller in each scenario is chosen based on Bellman's principle. Another design study [1] chooses motor and engine sizes to satisfy gradeability and acceleration constraints, while the battery size is determined to provide a certain AER based on minimizing the cost of the powertrain. In [4][5] we see that varying the component sizes has a significant effect on fuel and electric energy consumption due to the contribution from component mass and the scaling of the component efficiency maps. Above studies do not simultaneously vary all the component sizes. Hence, results provide understanding about the effect of varying a particular component size on PHEV energy consumption, but do not guarantee the optimal combination of all component sizes. We perform design optimization by varying all the component sizes simultaneously and finding an optimal combination for desired objectives.

It should be noted that most of the previous studies [1] [4] [6] consider a parallel or power-split architecture. Recently there has been a significant interest in the series architecture [2] [7] due to its relative simplicity and ease of achieving pure electric driving range. Larger batteries along with larger motors make it possible to provide more electric power and achieve higher displacement of fuel used by the engine. However, cost is a concern and this provides impetus for our study of optimal component sizing in a series electric propulsion system.

The design of a PHEV has to meet certain energy and power requirements. The ability of the PHEV to draw energy from the grid and the capacity of on-board storage enable it to meet a large portion of the road power demands with electric only operation. Therefore, the PHEV system performance is more sensitive to driving cycle selection than an HEV or a conventional vehicle. The energy and peak power demands depend on the type of driving, i.e. distance, type of road, and prevailing traffic conditions. In particular, driving patterns vary significantly with range, i.e. short distances are likely to be driven in the city, while long commutes typically involve significant portion of high-speed driving on freeways. Kwon et al. [8] examine the impact of certification drive cycles on component size requirements in a parallel PHEV and show that if the battery and electrical components are designed to have 40 miles of electric range on the UDDS, the same powertrain would have only 25 miles of electric range on the US06 cycle. A recent study by Tate at al. [7] recognizes the effect of real-world driving on design requirements and uses driving data collected in Southern California for assessing vehicle energy usage. Our previous study [5] indicated higher peak power and a much wider distribution of power demands in naturalistic cycles recorded in South East Michigan than federal cycles. Simulation analysis indicated strong dependency of PHEV CD range and fuel economy in CS mode on driving schedules [5], and established design constraints suitable for analysis under real-world conditions. Therefore, we bring real-world driving into this study rather than using customary repetitions of the federal urban driving schedule (UDDS) to maximize the relevance of findings.

The performance of PHEVs depends on control strategies too. Optimal controllers for different objectives, such as fuel and electricity cost are investigated in [4] [6]. In [9] a rule based controller is used to decide about switching between electric and gasoline power sources. Our study relies on a rule-based controller too, with the power thresholds being adjusted to account for battery scaling. While the controller may not represent the optimal benchmark, it is feasible and robust for system design studies.

The problem of optimizing the design (component sizes) of series electric PHEV for real world driving scenarios is addressed by coupling a predictive system simulation tool with an optimization framework. A virtual model of the series configuration with the ability to scale components is constructed in SIMULINK. Driving cycles deemed to be representative of real world driving for a given distance are used as inputs. Several references for algorithms that could be used for powertrain optimization are evaluated in [10]. It is argued that, even though gradient based algorithms like NLSQP are very fast and effective, they do not guarantee a global 
optimum. Due to the rule based nature of the controller and non-linear behavior of the component models it was observed that the objective function of energy consumption is not smooth and has multiple minima. In [11] these problems are clearly explained and the alternative of using a non-gradient based algorithm is suggested. Gao et al [12] discuss different non-gradient algorithms and explain the pros and cons. They also suggest that gradient based algorithms can be used along with non-gradient based algorithms such as DIRECT to refine the solution. Since we are interested in the trend produced by the optimal solutions the non-gradient DIRECT algorithm is deemed to be the best and sufficient for the task. The implementation uncovers the impact of the peak power and specific energy per mile on design decisions. The energy/mile of the drive cycle varies significantly with distance and leads to non-linear trends, particularly related to battery size.

The paper is organized as follows. The series hybrid electric powertrain configuration is described first. The analysis of naturalistic drive cycles is explained next, as well as the procedure for generating synthetic cycles representative of driving over a given distance. These driving cycles are used for evaluating the cost function during optimization runs. Explanation of the algorithm (DIRECT) and the optimization framework follows. The definition of the objective function allows the algorithm to produce the best design for a desired all-electric range of the PHEV. The results of the optimization are presented as tradeoffs that are required to achieve desired PHEV attributes.

\section{SERIES ELECTRIC POWERTRAIN}

The series hybrid configuration comprises of at least three machines: one engine, one electric generator, and one electric traction motor/generator. In Figure 1 the black solid lines show mechanical energy flow while the thin orange lines indicate flow of electrical energy. The supervisory controller sends signals to the engine, generator and the motor based on driver demand and vehicle system states and it is omitted in the schematic for simplicity. In our model the engine does not power the motor directly and all the energy produced by the engine is used to charge the battery, which in turn powers the motor. Since the battery and motor unit power the wheels, the driving motor has to be sized for the maximum road power requirements. The generator is sized in comparison to the engine rating and the state of charge (SOC) requirements during extended driving.

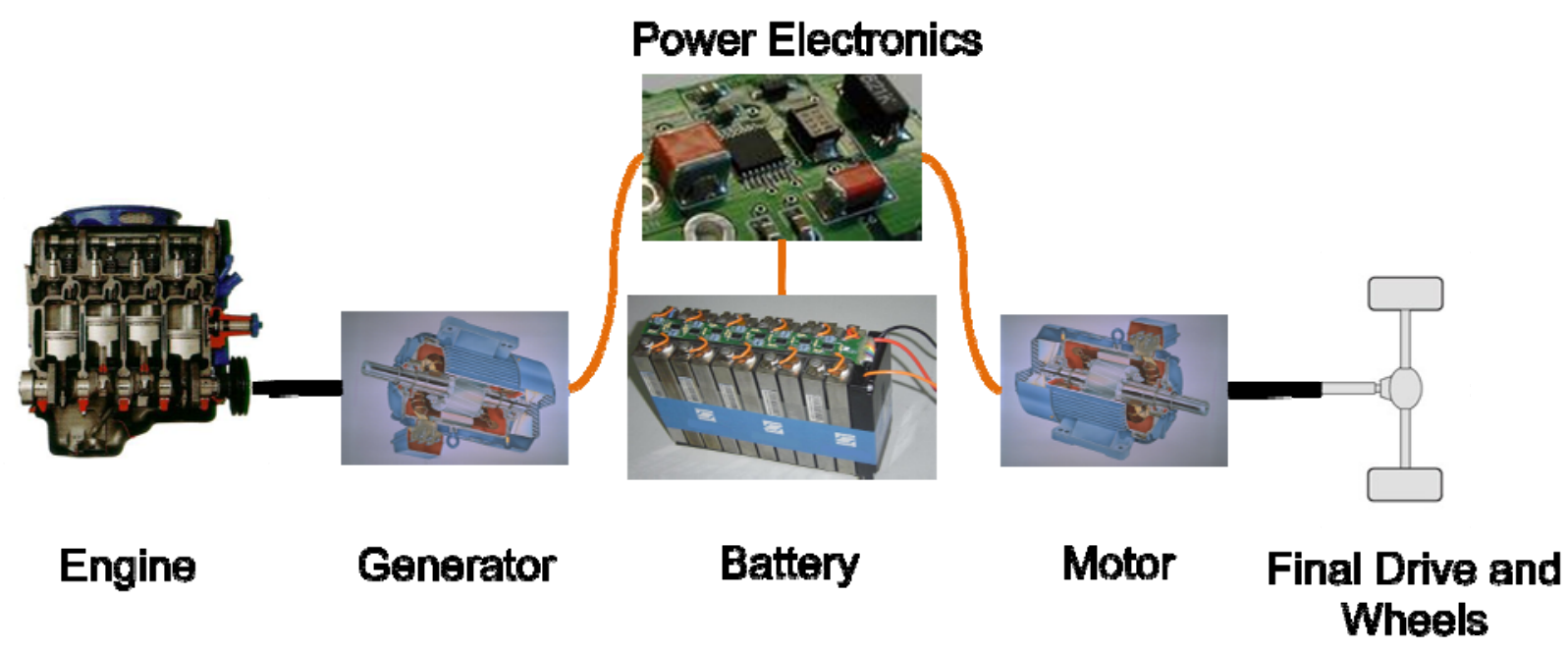

Figure 1. Schematic of the energy flow in a series hybrid electric configuration

PSAT [13], a forward-looking powertrain modeling platform developed in MATLAB-SIMULINK was used to model a representative series electric hybrid configuration. The simulation was created by choosing each component and integrating it into a typical architecture shown in Fig. 1. The component sizes and the control Page 3 of 14 
strategy were chosen to be similar to what is expected in the upcoming Chevy Volt. The intention was to provide a tool that allows studies of interactions in the series system and establishes a representative baseline for optimization, rather than to try to emulate the proprietary vehicle. The selected component sizes are given in table A1 in the Appendix.

\section{REAL-WORLD DRIVE CYCLE ANALYSIS}

University of Michigan Transportation Research Institute (UMTRI) conducted Field Operational Tests over the past several years to promote development of various vehicle safety devices and systems and to assess driver behavior [14]. Databases were created using a fleet of 11 mid-size sedans, equipped with data acquisition systems capable of recording over 200 channels of data. The time-speed traces were of particular interest here. A sample of 221 "trips" was obtained from the above mentioned database. Each trip starts when the key is placed in the ignition and ends when the key is taken out of the ignition. The trips cover a wide variety of driving distances and styles with a mixture of urban, suburban and highway driving. The naturalistic drive cycles have higher maximum velocities and lower stops/mile compared to certification cycles typically used for fuel economy estimation [5]. Our optimization technique requires a drive cycle for a given distance, but using individual naturalistic driving schedules directly would lead to solutions that depend too much on the personal driving style. Instead, we utilize the naturalistic database to create representative synthetic driving cycles suitable for implementation in the optimization framework. The characteristics of a set of naturalistic cycles are captured with a transition probability matrix that leads to creation of a Markov chain. Multiple synthetic cycles can be created using this technique, and we chose the one characterized with the specific energy (energy/mile) that matches the average of the initial set of naturalistic cycles.

\section{Procedure for obtaining Synthetic Drive Cycles}

The steps used in creating synthetic drive cycles and their description are as follows. Step 1 - Select a set of naturalistic drive cycles. The goal is to group representative drive cycles for $5,10,15,20,25,30,35$ and 40 mile lengths, hence we select naturalistic driving cycles with rounded distances within 1 mile of the target (i.e. 23.5 mile to 26.5 mile for the 25 mile cycle and so on). Step 2 - Use PSAT simulations of the base vehicle to determine specific energy for the selected cycles and calculate an average value. Step 3 - Create 50 synthetic drive cycles by using selected naturalistic drive cycle data from step 1. Consider Velocity and Road Power demand per unit vehicle mass as states. In every case chose the initial guess for the states randomly from the set of initial states of the selected naturalistic cycles. Generate a state transition matrix from the cycles in step 1 and create a Markov chain (drive cycle) with the restriction that the cycle length is within 1 mile window of the target distance required for the particular optimization case. Step 4 - Verify the representativeness of synthetic cycles by simulating PHEV performance and comparing their specific energy value to the average specific energy of the cycles selected in step 1. Figure 2 shows a plot of the specific energy consumption determined using the naturalistic cycles selected in step 1 and the 50 synthetic cycles generated for a 15 mile distance. Figure A1 in the Appendix shows all synthetic drive cycles used in the optimization study.

Page 4 of 14 


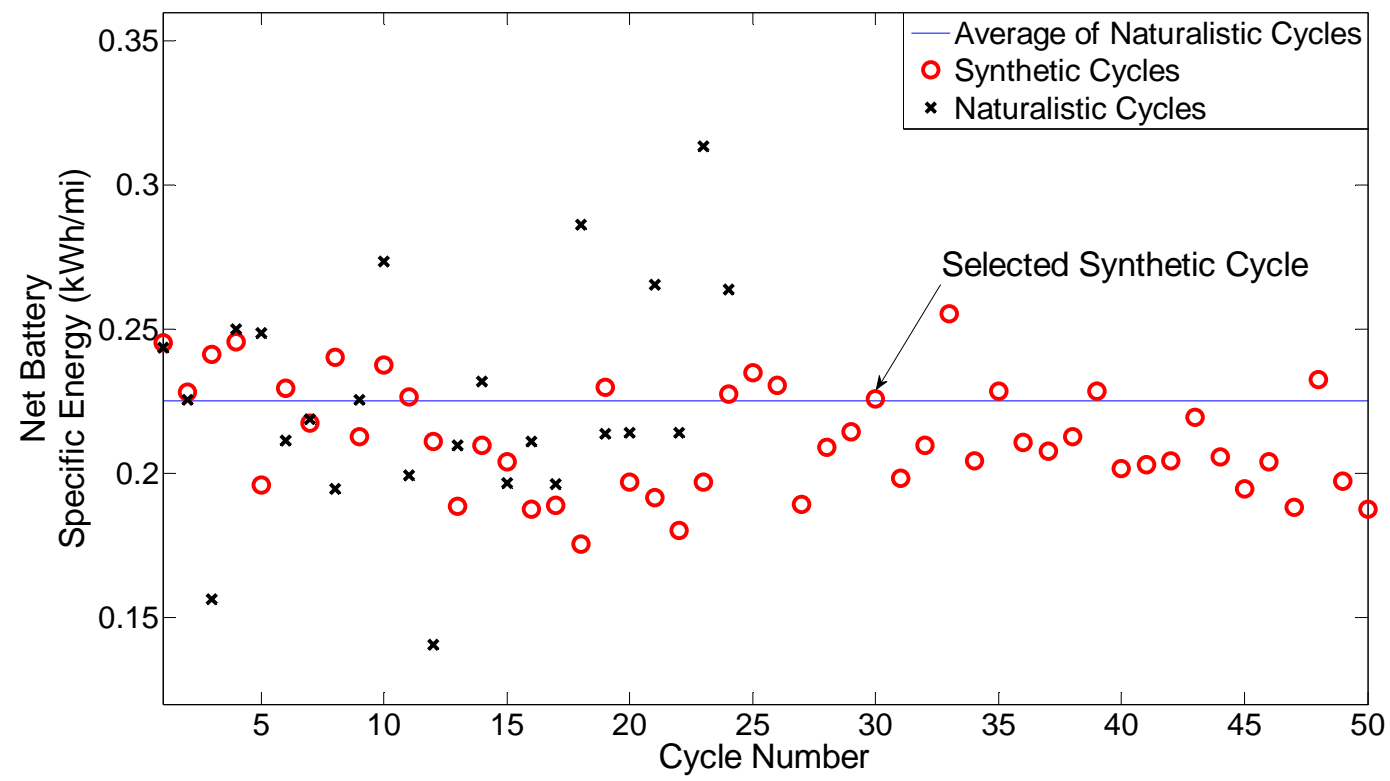

Figure 2. Specific Energies of Naturalistic and Synthetic Cycles (15 mile range)

\section{DESIGN OPTIMIZATION}

\section{Procedure}

The objective of our optimization study is to obtain a series electric powertrain design which minimizes the battery energy usage $\left(E_{b, \text { out }}\right)$ to achieve a desired AER. The battery discharging power limit $\left(P_{b, \text { disch } \max }\right)$ at the CS SOC of 0.4 and the maximum motor power $\left(P_{m \text {,max }}\right)$ are constrained (lower bound) to ensure that the vehicle has enough power to track the desired drive cycle. $P_{d m d \text {, max }}$ represents the maximum road power demand, though it is not equal to the exact maximum value. When comparing the battery and motor power capability to the maximum road power demand, component inefficiencies must be considered. In previous studies [5] the motor operating efficiencies ranged between $85-90 \%$ and the battery operating efficiencies ranged between $95-98 \%$. Hence we chose a factor of safety of 0.7 , which means that the value of $P_{d m d \text {,max }}$ is the maximum road power demand divided by 0.7 . The constraint on the SOC at the end of the cycle $\left(S O C_{\text {end }}\right)$ is to make sure that the battery discharges close to the charge sustaining SOC. This constraint (g3) along with the constraint on the number of engine on events (g5) ensures that we are finding the smallest battery that satisfies the objectives and constraints. The design variables $x_{m}$ and $x_{b}$ represent motor and battery sizes respectively and are scaled linearly to be within the 0 to 1 range. The constraint for engine-on events also ensures that we achieve a true AER, since that is a highly desired attribute for the series electric configuration. Since DIRECT requires inequality constraints in the actual optimization we modify the g5 by allowing less than two engine-on events. Since the engine size does not directly affect decisions about component sizes critical for All Electric operation [5], it is not considered as a variable and we make a decision based on the pre-optimality study described in the next paragraph. The problem formulation can mathematically be summarized as shown in Figure 3.

The solution to the above problem gives us the optimal battery and motor size to achieve a desired AER. It was seen in previous studies [5] that the effect of battery size variation on MPG in CS mode for a series PHEV is negligible compared to the effect of engine size (Figure 4). Due to this insensitivity of battery and motor size [5] Page 5 of 14 
to MPG, the engine size was chosen in a pre-optimality study as follows: simulate the powertrain with optimal battery and motor size starting with SOC of 0.45 where 0.4 is the CS SOC, vary engine sizes from $35 \mathrm{~kW}$ to $110 \mathrm{~kW}$ with increments of $1 \mathrm{~kW}$. The minimum size of $35 \mathrm{~kW}$ is chosen to make sure that the battery SOC is sustained at the threshold level in all the driving scenarios considered. A maximum of $110 \mathrm{~kW}$ is chosen for the engine size in order to satisfy the maximum road power demand of the drive cycles $(\sim 90 \mathrm{~kW})$ considering the drivetrain losses. Finally, chose the engine size which produces the highest MPG.

$$
\begin{gathered}
\min _{x_{m}, x_{b}} E_{b, \text { out }} \\
\text { subject to: } \\
P_{b, \text { disch_max }}(S O C=0.4) \geq P_{d m d \text {,max }} \\
P_{m, \text { max }} \geq P_{d m d, \text { max }} \\
0.37 \leq S O C_{\text {end }} \leq 0.43 \\
\quad x_{m}, x_{b} \in[0,1] \\
\text { engineon event }=0
\end{gathered}
$$

Figure 3. Mathematical formulation of Optimization problem

\section{Algorithm}

The battery energy usage in a PHEV depends on variables, such as component sizes, and control strategy parameters. Due to the non-linear nature of component efficiency and resistance maps, as well as the switching nature of the rule based controller the objective function was found to be not smooth (not differentiable) and not continuous (Figure 5). Gradient based algorithms such as Sequential Quadratic Programming (SQP) use the derivative information to find the optimum. Therefore, the decision based on derivatives could be a local minimum rather that a globally optimal solution. Non-gradient based algorithms such as DIRECT, Simulated Annealing (SA), Genetic Algorithm (GA), and Particle Swarm Optimization (PSO) do not rely on gradients, and therefore work well when the objective function is discontinuous [11]. Our problem requires such an algorithm and we opted for DIRECT.

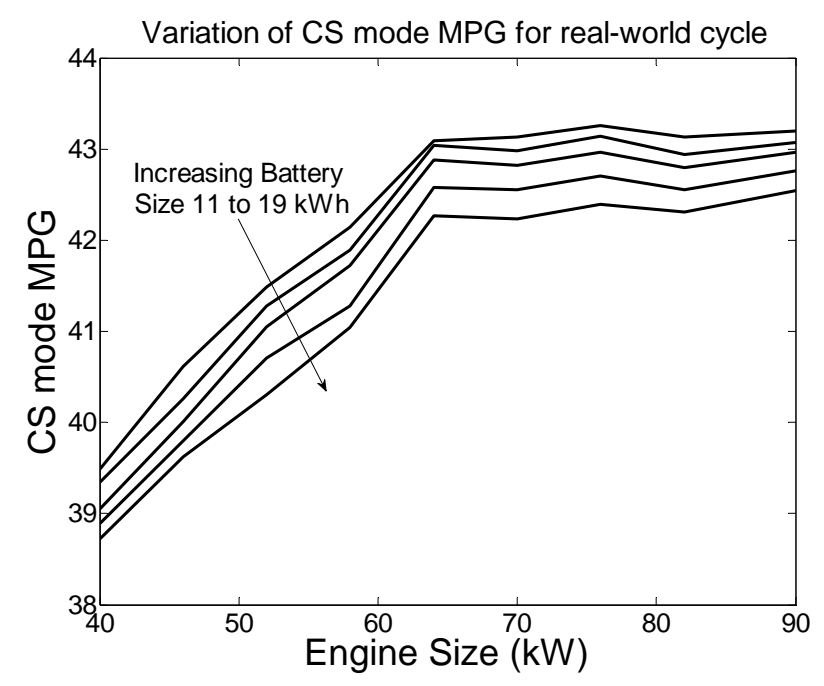

a)

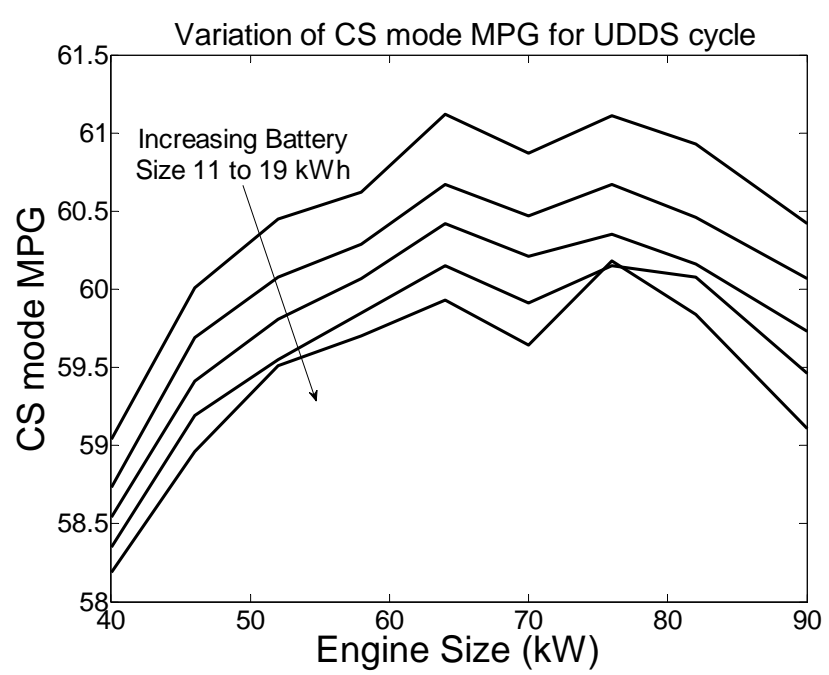

b)

Figure 4. MPG variation in CS mode with Engine and Battery Size for a) real-world suburban drive cycle b) UDDS

Page 6 of 14 


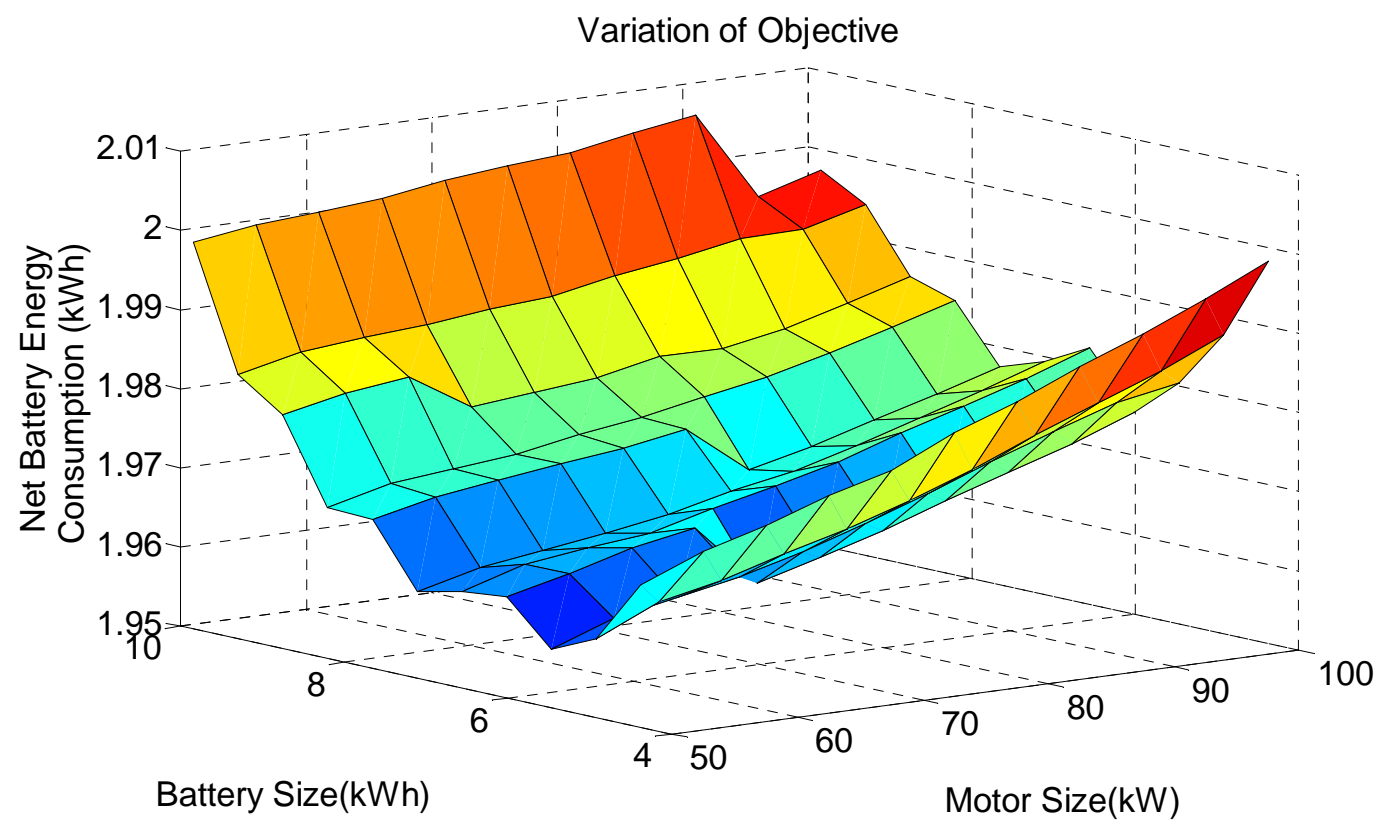

Figure 5. Battery Energy Consumption as a function of design variables.

DIRECT is a sampling algorithm, developed by Donald R. Jones [15]. The name comes from shortening the phrase "DIviding RECTangles", which describes the way the algorithm moves towards the optimum. The DIRECT method has been shown to be very competitive with other algorithms in its class [15][16]. The strengths of DIRECT lie in the balanced effort given to local and global searches, and the relatively few parameters required to run it.

\section{RESULTS AND DISCUSSION}

As discussed previously, the optimization problem was decoupled into finding the optimal battery and motor sizes for a desired AER, and subsequently determining the corresponding engine size based on analysis of CS operation. Fourteen optimization runs were performed for different driving scenarios with battery and motor size as design variables and the net battery energy usage as the cost function. Before discussing the results, it is important to understand the effect of constraints on the optimal decisions. As previously discussed g1 and g2 are power constraints, g3 is a constraint on the SOC at the end of the simulation and g5 is the constraint on number of engine-on events, which together translate to a battery energy capacity constraint. g4 is a set constraint which is necessary to specify an upper and lower bound on the design variables to define the search space for the DIRECT algorithm.

If the search space defined by constraint g4 contains a feasible region, then the battery size variable $x_{b}^{*}$ is affected by constraints g1, g3 and g5. The g1 indicates that the optimal battery size should be capable of providing a certain desired amount of power at its lowest operating SOC, while g3 and g5 together say that the battery should contain enough energy to complete the trip. Equation 1 below describes the maximum battery discharging power capability (in $\mathrm{kW}$ ) as a function of battery size [13].

$$
P_{b, \text { disch_max }}(S O C=0.4)=\frac{206.41}{6 * 3.6} * \text { Battery_Size }(k W h)
$$


For short synthetic cycles, e.g. 5 and 10 mile distance ranges, the $P_{d m d \text {,max }}$ value is $79.95 \mathrm{~kW}$ and $66.68 \mathrm{~kW}$ respectively. It should be noted that the longer cycle (10 mi), has a lower maximum power demand value than the shorter cycle (5 mi). From 1 , if we set $P_{b \text {,disch_max }}(S O C=0.4)$ as $P_{d m d \text {,max }}$ (making constraint g1 active) we get the minimum battery size required to satisfy g1 as $8.36 \mathrm{kWh}$ for $5 \mathrm{mi}$ cycle and $6.98 \mathrm{kWh}$ for $10 \mathrm{mi}$ cycle. Constraint g2 affects the choice of optimal motor size $x_{m}^{*}$. We choose a motor size that satisfies g2 as an equality and that leads to the smallest motor size. Simulating the powertrain model with the above battery and motor sizes produces results shown in Figure 6 (a) and (b).

In both cases constraint g3 is violated and the battery has more energy than required to complete the driving task. Choosing a battery size smaller than $8.36 \mathrm{kWh}$ for $5 \mathrm{mi}$ cycle and $6.98 \mathrm{kWh}$ for 10 mi cycle would result in insufficient power to complete the drive cycle. Thus, the above values of battery and motor sizes are chosen, but with the understanding that they are oversized in terms of energy requirements. Indeed, Fig. 6 shows a significant amount of unused battery energy at the end of the desired travel distance. Similar analysis was adopted to obtain the battery and motor size in other cases where the power constraint (g1) was active. In cases where power constraint is not active, the battery size is determined by its energy capacity, i.e. constraint g3 is active and g5 is satisfied, and we obtain optima for both battery and motor sizes directly from the algorithm. As an example, simulating the powertrain over the 25 and 30 mile cycles makes the power constraint (g1) inactive and leads to the pattern for SOC variation shown in Figure 7 (a) and (b).

Table A2 in the Appendix gives a detailed description of the results for all cases, including the number of function evaluations required to get the solution. In cases where the amount of energy used is less than the usable battery energy, it is due to the power constraint (g1) activity. If all the usable battery energy is consumed the energy constraint becomes active. Figure 8 shows the effect of these constraints on Optimal Battery Size. For the cases where the power constraint is not active the algorithm produces a true optimum for battery and motor size. This optimization procedure was stopped when there was no change in the objective function for 100 function evaluations.

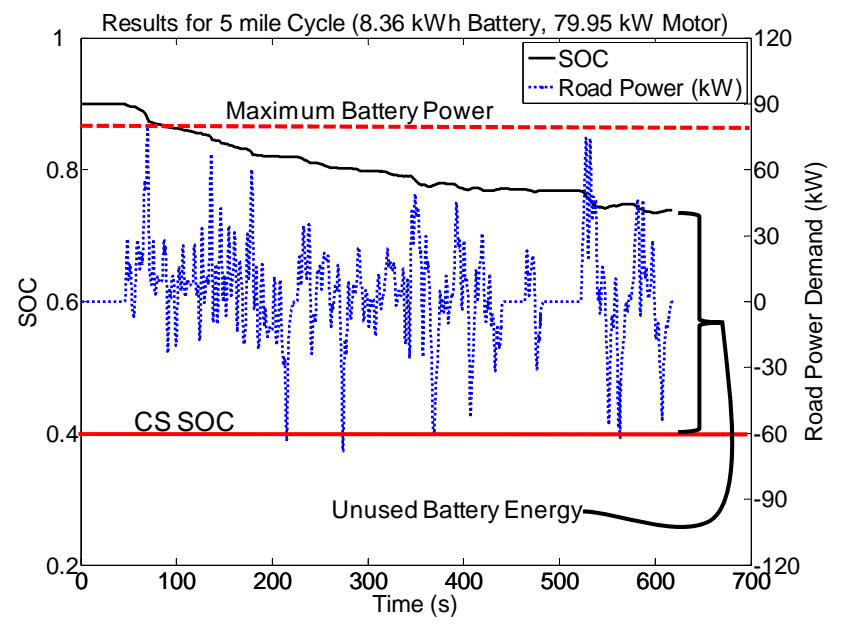

a)

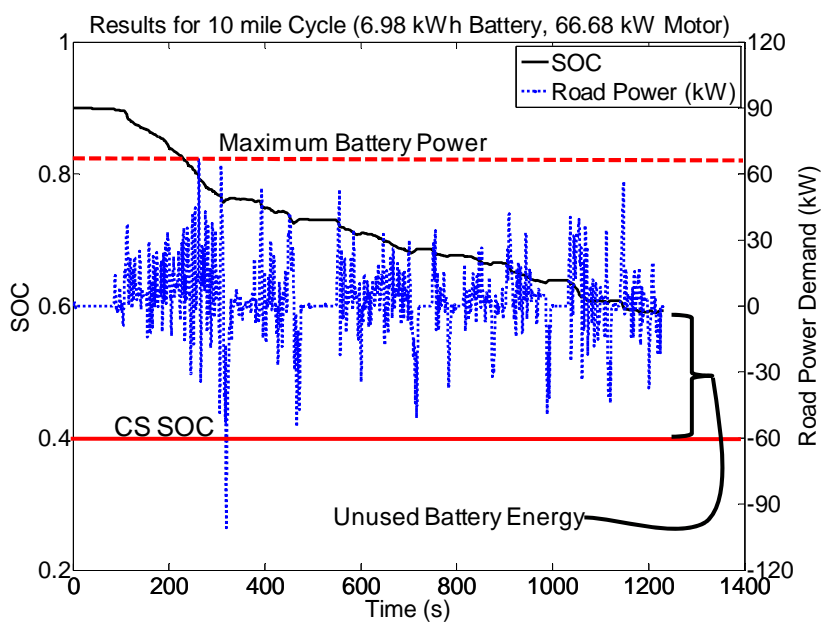

b)

Figure 6. SOC variation over different drive cycles with optimal component sizes a) 5 mile cycle b) 10 mile cycle. 


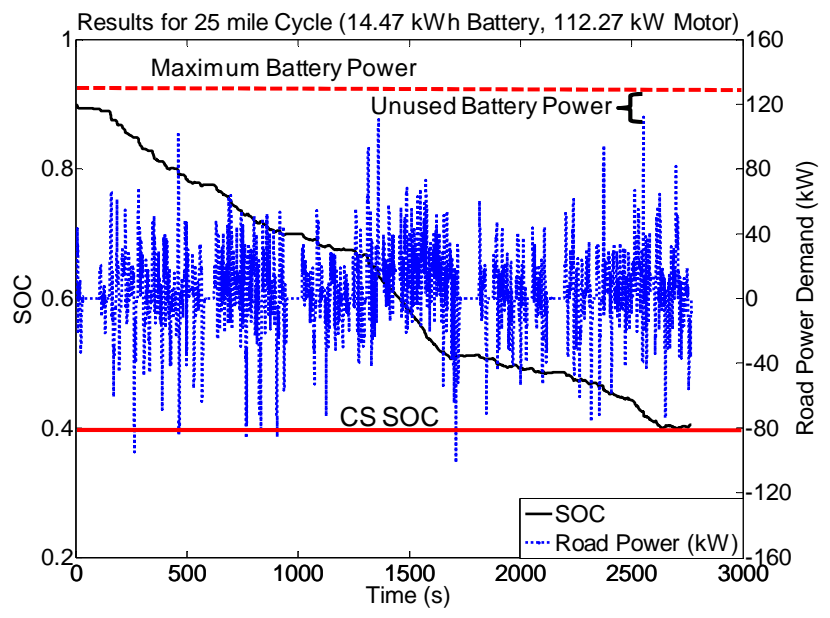

a)

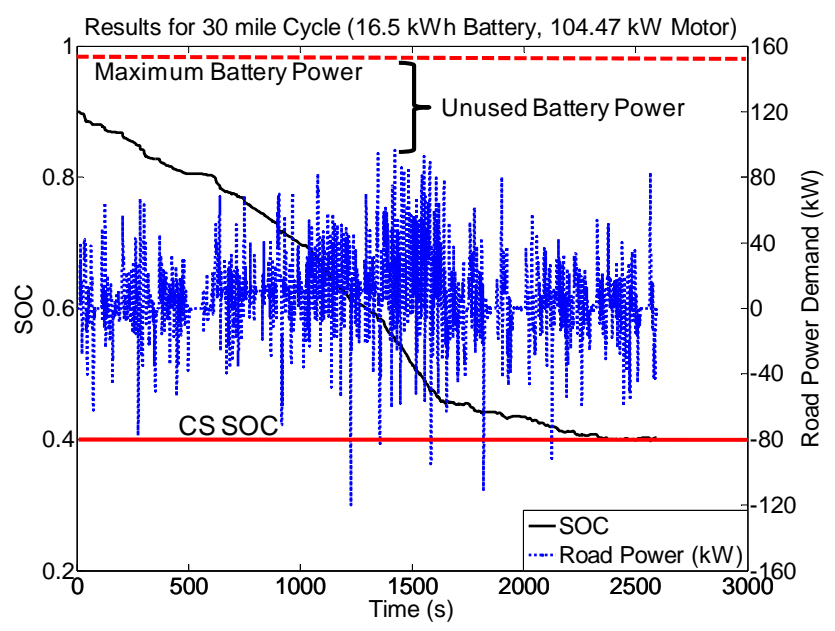

b)

Figure 7. SOC variation over different drive cycles with optimal component sizes a) 25 mile cycle b) 30 mile cycle.

It was seen that in all cases the smallest motor size that satisfies the constraint g2 is the optimal motor size. As motor size increases it contributes to an increase in vehicle mass and the expansion of the motor efficiency maps result in a decrease of overall energy efficiency. Hence the optimum represents a smallest allowable motor size, as desired and in agreement with findings in [5].

In Figure 9a, we observe that there is a significant difference in the required optimal battery size for a particular desired AER if we consider real-world driving rather than certification urban cycle FTP75 (also called UDDS). There are 3 major observations from Figure 9a, i.e.: (i) The optimal battery energy values are higher for the naturalistic case compared to UDDS, suggesting that sizing the battery for UDDS would lead to underperformance in the real world, (ii) The abrupt changes of slope observed at low range (up to 20 miles for real-world and up to $\sim 10$ miles for UDDS) are due to power constraint activity. Since the vehicle power demands for UDDS are lower than real-world cycles, the power constraint is active over a shorter range; (iii) The overall shape of the curve obtained for real-world conditions is non-linear towards the high end, because longer commutes generally lead to more high-speed driving and accelerations at higher speeds. This translates into higher power demands and energy consumption. The specific energy numbers shown in table A2 are in general agreement with the results shown in a study by Tate et al. that considered a real world driving sample from Southern California [7]. In contrast, when repetitions of the UDDS cycle are used, the specific energy consumption remains almost constant regardless of the distance traveled, with nearly negligible variations due to increased battery mass. Consequently, the difference in optimal battery sizes calculated for real-world driving vs. UDDS becomes progressively higher. A comparison of the optimal motor sizes for real-world driving vs. UDDS in Figure 9b shows that the optimal motor size for the real world is significantly higher, being nearly twice as big in some cases. As there are only 8 points (motor sizes) for different real-world trips, the plot does not seem to show a trend, but the motor size is basically decided by the maximum road power demand which generally increases with distance. If a larger number of trip lengths were considered the optimal motor sizes would likely increase with distance initially and then taper off at longer distances as the maximum driving speeds are limited. The final decision should be made judiciously, after considering the tradeoff between the cost and importance of the pure AER operation.

Table A2 also shows the results for optimal engine size calculation. The instantaneous charge depletion in the battery is directly dependent on the road power demand, and the power demand from the engine is a function of 
the battery SOC. Hence, while the dependency is indirect, the higher road power demand leads to higher instantaneous battery charge depletion and higher power demanded from the engine.

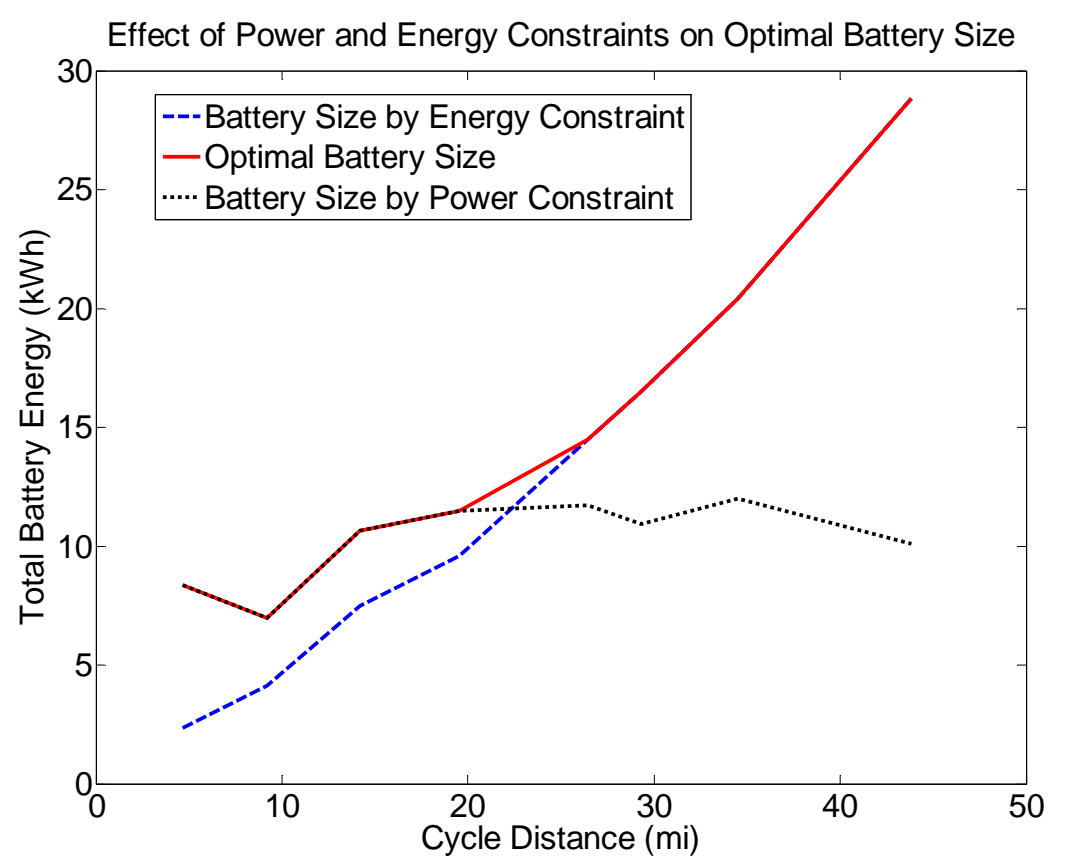

Figure 8. Effect of Constraints on Optimal Battery Size.

Engine operates most efficiently at high torque and medium speed. If the power demands are very high the engine is forced to operate at full load. The road power demands for the real-world drive cycles are higher than the road power demands for UDDS, but the engine is sized more generously too, hence large fraction of engine operation takes place near the optimum for real-world conditions. It is observed that MPG decreases with increasing drive cycle distance. This is mainly due to the increased battery and motor mass and increased energy consumption per mile. The MPG in 30s are relatively low for a hybrid, but this is mainly due the multiple energy conversions between the engine and the wheels and component design being driven by the AER objective.

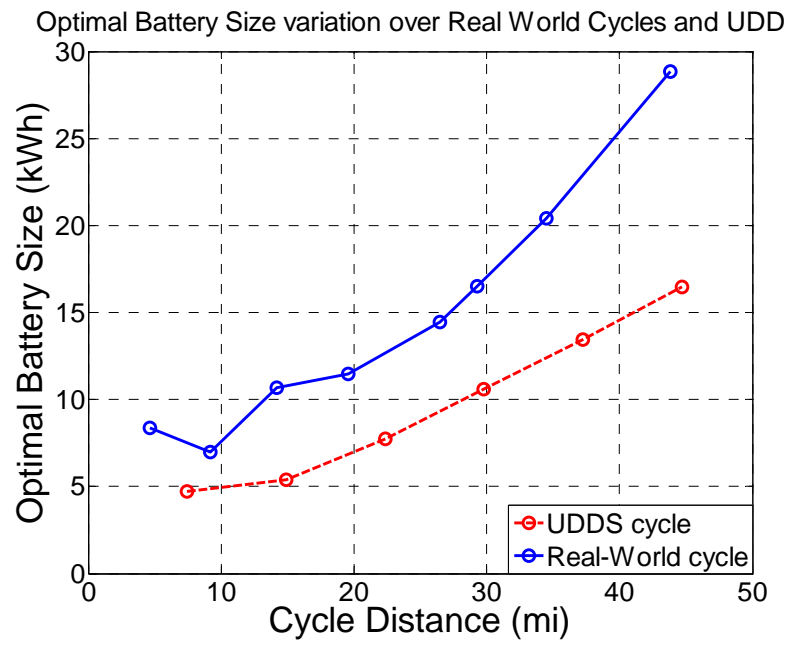

a)

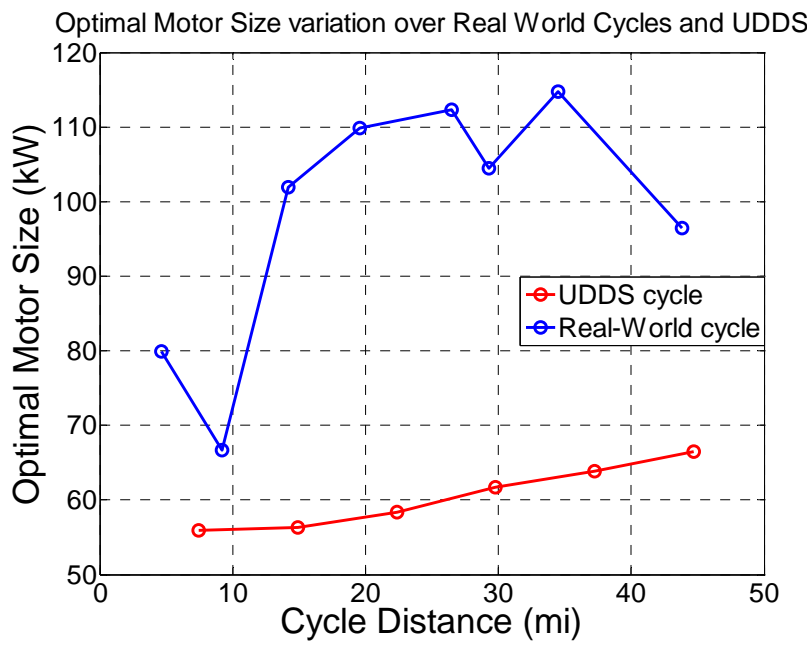

b)

Figure 9. Comparison of a) Optimal Battery and b) Optimal Motor Size for Real-World driving and UDDS. Page 10 of 14 


\section{CONCLUSIONS}

This paper proposes a framework to optimize the design of a series PHEV powertrain for desired All Electric Range (AER). A unique aspect of our approach is consideration of real-world driving in the optimization procedure. Real-World driving is characterized from a database of naturalistic driving generated in Field Operational Tests originally intended for evaluation of safety systems. The procedure utilizes Markov chains to generate synthetic drive cycles representative of real-world driving. Subsequently, PHEV optimization is performed in two steps. The optimal battery and motor sizes to achieve a desired All Electric Range (AER) with least amount of energy are determined first. A synthetic cycle representative of driving over a given range is used for function evaluations. Then, the optimal engine size is obtained by considering fuel economy in the charge sustaining (CS) mode.

The higher power/energy demands of real-world cycles lead to PHEV designs with substantially larger batteries and engines than those developed using repetitions of the federal urban cycle (UDDS). In particular:

- The battery size is governed by power constraints for short AERs, whereas the battery energy capacity is more important for longer AERs.

- The required optimal battery size increases non-linearly with desired AER, increasing more rapidly at longer desired AERs. The reason for this non-linearity is the higher speed driving characteristic of longer trips. High speed accelerations result in higher power demands than similar accelerations at lower speeds. This non-linearity in optimal battery size with desired AER when compared to the linear trend in optimal battery sizes produced for design based on UDDS cycles results in increasing difference in battery sizes with longer desired AERs.

- The findings indicate that designing a PHEV with longer desired AER would mean a greater difference in cost and performance between real-world and UDDS designs.

- The optimal engine was designed based on CS mode with MPG minimization objective. The effect of larger road power demands in real-world driving and larger electrical components cascades down and leads to a larger engine size too, although the effect is indirect and dampened.

In summary, the design of a series PHEV should be approached with clear objectives and priorities in mind. Considering real-world driving will enable much better understanding of tradeoffs in the light of consumer expectation and the impact on the grid. If the pure AER is deemed to be a critical attribute, using the real-world driving information is a necessity and will lead to designs much different than those based on repetitions of the certification schedule such as UDDS. The final decision should be made judiciously, as increased battery and motor size strongly affect the cost of the whole vehicle. In other words, a possibility of allowing a small number of engine-on events during the charge depleting operation rather than insisting on pure electric operation may provide an interesting tradeoff.

\section{ACKNOWLEDGEMENTS}

This work was funded by the Department of Energy as part of the program "Technical Challenges of Plug-In Hybrid Electric Vehicles and Impacts to the U.S. Power System“, subcontract \#46827. The authors would like to thank Zevi Baraket and Tim Gordon of UMTRI for providing the naturalistic driving data and for their valuable insights pertaining to driver behavior, and Michael Kintner-Meyer of Pacific Northwest National Laboratory for technical guidance provided during the course of the project.

Page 11 of 14 


\section{REFERENCES}

1. Sam Golbuff, "Design Optimization of a PHEV", SAE 2007-01-1545.

2. Vincent Freyermuth, Eric Fallas and Aymeric Rousseau, "Comparison of Powertrain Configuration for Plug-in HEVs from a Fuel Economy Perspective”, SAE 2008-01-0461.

3. Ahmad Pesaran, Tony Markel, Harshad Tataria, David Howell, "Battery Requirements for Plug in Hybrid Electric Vehicles - Analysis and Rationale", EVS-23, Anaheim, California, December, 2007.

4. Dominik Karbowski, Chris Haliburton, Aymeric Rousseau, "Impact of Component Size on PHEV Energy Consumption Using Global Optimization", EVS23, Anaheim, CA, Dec. 2007.

5. Rakesh Patil, Brian Adornato, Zoran Filipi, "Impact of Naturalistic Driving Patterns on PHEV Performance and System Design", SAE 2009-01-2715.

6. S. J. Moura, H. K. Fathy, D. S. Callaway, J. L Stein, "A Stochastic Optimal Control Approach for Power Management in Plug-in Hybrid Electric Vehicles," to be published in Proc. of 2008 Dynamic Systems and Controls Conference, Ann Arbor, 2008.

7. E.D.Tate, Michael O. Harpster and Peter J. Savagian, "The Electrification of the Automobile: From Conventional Hybrid, to Plug-in Hybrids, to Extended-Range Electric Vehicles”, SAE 2008-01-0458.

8. J. Kwon, J. Kim, E. Fallas, S. Pagerit and A. Rousseau "Impact of Drive Cycles on PHEV component requirements", , SAE 2008-01-1337.

9. Aymeric Rousseau, Sylvain Pagerit, David Gao, "Plug-in Hybrid Electric Vehicle Control Strategy Parameter Optimization", EVS-23, Anaheim, California, December, 2007.

10. Massimiliano Gobbi, Imtiaz Haque, Panos P.Y. Papalambros, Giampiero Mastinu, "A Critical Review of Optimization Methods for Road Vehicles Design", AIAA/ISSMO Multidisciplinary Analysis and Optimization Conference, September 2006, AIAA 2006-6998.

11. Ryan Fellini, Nestor Michelena, Panos Papalambros, and Michael Sasena, "Optimal Design of Automotive Hybrid Powertrain Systems", Environmentally Conscious Design and Inverse Manufacturing Proceedings, February 1999.

12. Wenzhong Gao, Sachin Kumar Porandla, "Design Optimization of a Parallel Hybrid Electric Powertrain", Vehicle Power and Propulsion, 2005 IEEE Conference.

13. http://www.transportation.anl.gov/modeling_simulation/PSAT/index.html

14. "Road Departure Crash Warning System Field Operational Test: Methodology and Results”, UMTRI, June 2006.

15. D. R. Jones et. al., "Lipschitzian Optimization without the Lipschitz Constant", Journal of Optimization Theory and Application, Vol 79, No 1, Oct 1993.

16. Daniel E. Finkel, "DIRECT optimization algorithm user guide", North Carolina State University, March 2003.

\section{CONTACT INFORMATION}

Zoran Filipi, filipi@umich.edu 


\section{APPENDIX}

\begin{tabular}{|l|l|}
\hline Component & Specifications \\
\hline Engine & 1 L Honda Insight VTEC-E gasoline \\
\hline Motor/Generators & $100 \mathrm{~kW}$ peak UQM PowerPhase PM motor \\
\hline Battery & $\begin{array}{l}\text { Li-ion, 6Ah 75 cell SAFT model scaled to } 16 \mathrm{kWh} \text { Total } \\
\text { Energy (50\% usable) }\end{array}$ \\
\hline Final Drive Ratio & 3.07 \\
\hline Resistance Coefficients & $\mathrm{f0}=88.6 \mathrm{~N}, \mathrm{f} 1=0.14 \mathrm{~N}-\mathrm{s} / \mathrm{m}, \mathrm{f} 2=0.4392 \mathrm{~N}-\mathrm{s}^{\wedge} 2 / \mathrm{m}^{\wedge} 2$ \\
\hline
\end{tabular}

Table A1 - Powertrain Model Specifications for Series Vehicle

\begin{tabular}{|l|l|l|l|l|l|l|l|l|l|}
\hline $\begin{array}{l}\text { Cycle } \\
\text { Name }\end{array}$ & $\begin{array}{l}\text { Cycle } \\
\text { Distance } \\
(\mathrm{mi})\end{array}$ & $\begin{array}{l}\text { Optimal } \\
\text { Total } \\
\text { Battery } \\
\text { Size } \\
\mathrm{kWh})\end{array}$ & $\begin{array}{l}\text { Optimal } \\
\text { Motor } \\
\text { Size } \\
\mathrm{kW})\end{array}$ & $\begin{array}{l}\text { Battery } \\
\text { Energy used/ } \\
\text { Optimization } \\
\text { Objective } \\
(\mathrm{kWh})\end{array}$ & $\begin{array}{l}\text { Usable } \\
\text { Battery } \\
\text { Energy } \\
\mathrm{kWh})\end{array}$ & $\begin{array}{l}\text { Specific } \\
\text { Battery } \\
\text { Energy } \\
\text { Consumption } \\
(\mathrm{kWh} / \mathrm{mi})\end{array}$ & $\begin{array}{l}\text { Function } \\
\text { Eval- } \\
\text { uations }\end{array}$ & $\begin{array}{l}\text { Optimal } \\
\text { Engine } \\
\text { Size } \\
(\mathrm{kW})\end{array}$ & MPG \\
\hline 5 mile & 4.65 & 8.37 & 79.95 & 1.113 & 4.057 & 0.239 & & 87 & 63.95 \\
\hline 10 mile & 9.17 & 6.98 & 66.68 & 1.966 & 3.384 & 0.214 & & 89 & 49.54 \\
\hline 15 mile & 14.19 & 10.67 & 101.93 & 3.551 & 5.173 & 0.250 & & 82 & 41.87 \\
\hline 20 mile & 19.55 & 11.49 & 109.84 & 4.620 & 5.575 & 0.236 & & 102 & 44.41 \\
\hline 25 mile & 26.46 & 14.47 & 112.27 & 7.021 & 7.021 & 0.265 & 227 & 97 & 38.27 \\
\hline 30 mile & 29.29 & 16.51 & 104.47 & 8.063 & 8.063 & 0.275 & 523 & 103 & 37.23 \\
\hline 35 mile & 34.48 & 20.42 & 114.81 & 9.952 & 9.952 & 0.289 & 153 & 96 & 36.29 \\
\hline 40 mile & 43.83 & 28.83 & 96.47 & 13.924 & 13.924 & 0.318 & 439 & 110 & 33.28 \\
\hline $1 \mathrm{x}$ udds & 7.45 & 4.71 & 55.93 & 1.353 & 2.284 & 0.181 & & 50 & 73.01 \\
\hline $2 \mathrm{x}$ udds & 14.9 & 5.38 & 56.29 & 2.629 & 2.629 & 0.176 & 213 & 51 & 61.76 \\
\hline $3 \mathrm{x}$ udds & 22.35 & 7.75 & 58.39 & 3.825 & 3.825 & 0.171 & 265 & 67 & 60.03 \\
\hline 4x udds & 29.8 & 10.58 & 61.69 & 5.182 & 5.182 & 0.174 & 221 & 67 & 59.36 \\
\hline $5 x$ udds & 37.25 & 13.45 & 63.83 & 6.561 & 6.561 & 0.176 & 431 & 67 & 58.93 \\
\hline $6 x$ udds & 44.7 & 16.45 & 66.50 & 8.003 & 8.003 & 0.179 & 491 & 67 & 58.67 \\
\hline
\end{tabular}

Table A2 - Design Optimization Results for different driving scenarios 

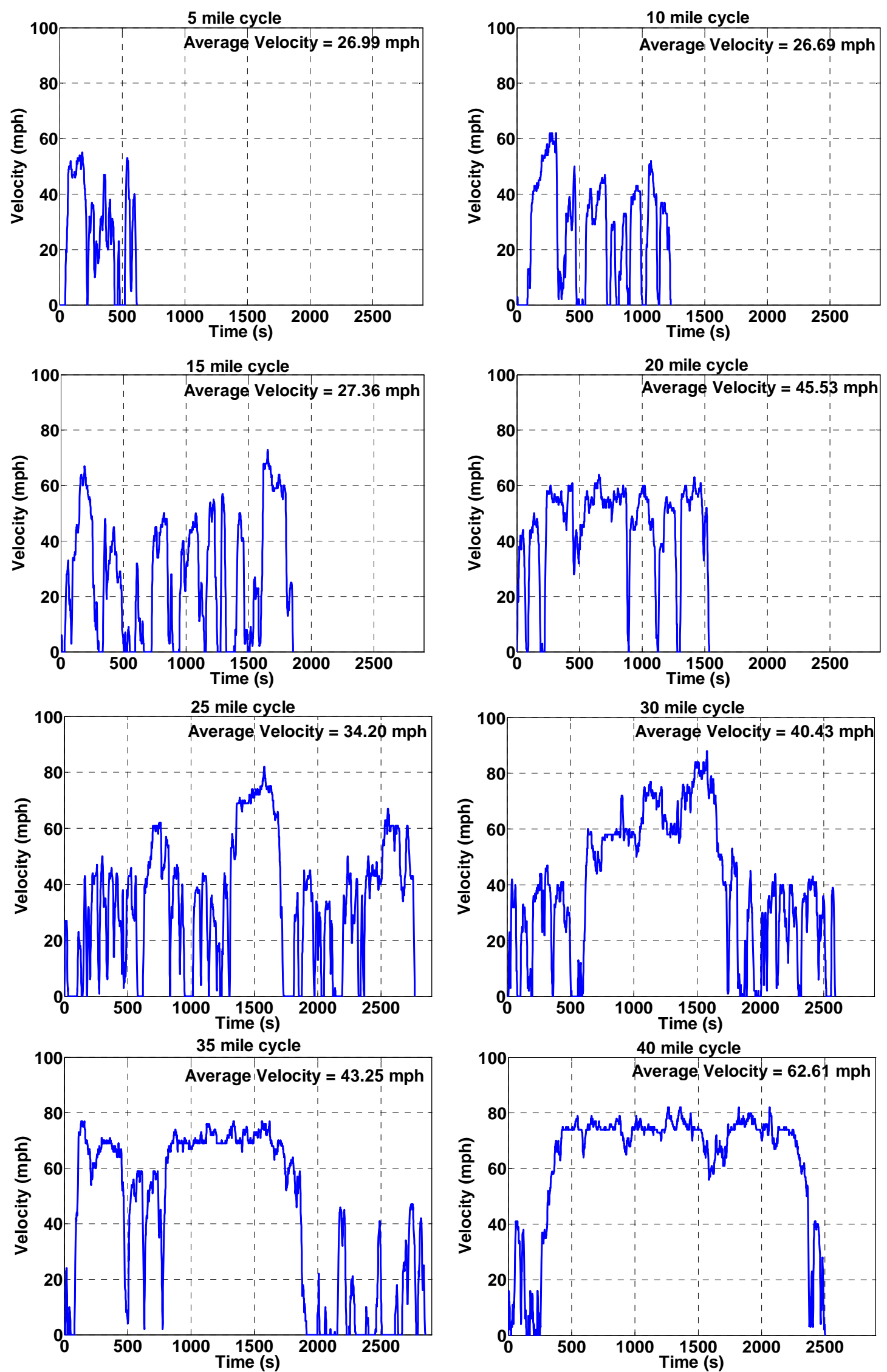

Figure A1. Synthetic Drive Cycles (5 miles to 40 miles length)

Page 14 of 14 